\title{
Weight Estimation of the Sea Cucumber (Stichopus japonicas) using Vision-based Volume Measurement
}

\author{
Donggil Lee*, Seonghoon Kim*, Miseon Park** and Yongsu Yang ${ }^{\dagger}$
}

\begin{abstract}
Growth analysis and selection of sea cucumbers (Stichopus japonicas) is typically performed through length or weight measurements. However, because sea cucumbers continuously change shape depending on the external environment, weight measurement has been the preferred approach. Weight measurements require extensive time and labor, moreover it is often difficult to accurately weigh sea cucumbers because of their wet surface. The present study measured sea cucumber features, including the body length, width, and thickness, by using a vision system and regression analysis to generate $R^{2}$ values that were used to develop a weight estimation algorithm. The $R^{2}$ value between the actual volume and weight of the sea cucumbers was 0.999 , which was relatively high. Evaluation of the performance of this algorithm using cross-validation showed that the root mean square error and worst-case prediction error were $1.434 \mathrm{~g}$ and $\pm 5.879 \mathrm{~g}$, respectively. In addition, the present study confirmed that the proposed weight estimation algorithm and single slide rail device for weight measurement can measure weights at approximately 4,500 sea cucumbers per hour.
\end{abstract}

Keywords: Sea cucumber, Weight, Volume, Vision system

\section{Introduction}

The lengths and weights of marine animals are typically measured to determine growth and to select individuals. Weight measurements by scale are the most popular approach [1]. The weight measurement method requires frequent calibrations - i.e., taring the scale - and often hinders accurate and rapid measurement because of the webbed surface of the measured object.

To improve these methods, previous studies measured the lengths [2-5] and areas [6-7] of fish in two-dimensional images generated by a computer vision system; fish selection and weight estimation were then conducted. Lee et al. (2001) performed three-dimensional measurements and restructured a two-dimensional cross-section photography scan into a three-dimensional surface using charge coupled device (CCD) cameras and a multi-line laser [8-9]. In addition, it is possible to calculate the area of an oyster body using the cubic splines method [10] to estimate weight. However, unlike other marine animals, the sea cucumber (Stichopus japonicas) changes its shape into a football-like [11] or globular structure when it shrinks [12]; moreover, its shape is irregular when it is fully extended. Thus, it is difficult to apply the existing method of weight estimation by measuring the body length $(B L)$, body width

$\uparrow$ Corresponding Author: Fisheries System Engineering Division, National Fisheries Research \& Development Institute, Korea. (ysyang@korea.kr)

* Fisheries System Engineering Division, National Fisheries Research \& Development Institute, Korea. (dongsoon@nfrdi.go.kr)

** Aquaculture Management Division, National Fisheries Research \& Development Institute, Korea. (oysterking@korea.kr)

Received: April 24, 2014; Accepted: August 13, 2014
$(B W)$, thickness $(T)$, and view area [13]. In Japan, the body size of the sea cucumber is measured after the administration of $l$-menthol anesthesia. However, because this method cannot be easily applied at practical sites, Yamana and Hamano derived a regression equation that uses a digital still camera to measure the $B L$ and $B W$ of the sea cucumber at any given time; it then converts the $B L$ and $B W$ to the image that is equivalent to its anesthetized condition [14]. Yingst formulated the size index $(\mathrm{SI})=B L \times B W$ to determine body size of the sea cucumber [15]. Moreover, Mitsukuri proposed an equation that facilitates calculation of the sea cucumber's body size using its square length and width [16]. However, the above calculation methods are inaccurate because they include no information on the thickness of the sea cucumber; rather, they are simply based on observations of shape changes in which the animal gradually becomes slender, during its extension. Body volume of the animal seems to be relatively stable during body extension; however, the influence of water storage in its respiratory tree remains to be clarified [14].

Existing measurement methods using sea cucumber volume or weight are impractical when a large quantity of farm-raised sea cucumbers are to be measured, particularly in a short time period; a substantial amount of time and labor are required for accurate measurements. Therefore, to generalize their ever-changing shapes, quickly and accurately analyze their growth or select them at any given time, a technology that automatically estimates sea cucumber weight is required.

The purpose of this study was to develop an algorithm that estimates sea cucumber weight by measuring its 
features, which include $B L, B W, T$, and volume, using the image processing technology of vision system. To simply perform the measurement of the sea cucumber — instead of using the existing method of measuring its $B L$ and $B W$ - it was assumed that the early shape of the sea cucumber in air is a half oblate ellipsoid. Therefore, the volume of the sea cucumber was measured using the $B L, B W$, and $T$, which were measured at the time of sample, and by using the volume equation for a modified ellipsoid. Accordingly, regression analyses of the calculated and actual volumes of sea cucumbers were performed for optimization. For optimal design of the weight estimation algorithm, regression analysis was conducted on each feature of the sea cucumbers against the weight, and the $R^{2}$ value and regression equation were examined. To confirm the validity and performance of the final weight estimation algorithm, replicates were performed using the single slider rail, which was produced in a laboratory-sized system for the present study.

\section{Material and Methods}

\subsection{Experimental animals: Sea cucumeber}

In January 2014, 350 sea cucumbers (weight range: 0.23-92.56 g) were collected by divers from the southern coast of Korea. The depth of the sampling location did not exceed $11 \mathrm{~m}$ and the seawater temperature at the time of sampling was $13.8{ }^{\circ} \mathrm{C}$. The sea cucumbers were transferred to the nearby laboratory of the Aquaculture Management Division and maintained in a tank, which was equipped with flowing $100 \mu \mathrm{m}$ filtered seawater at ambient temperature $\left(14{ }^{\circ} \mathrm{C}\right)$. All animals were starved for $24 \mathrm{~h}$ before the experiment to minimize the effect of excretion on weight.

\subsection{Measurement of actual volume and weight}

Actual volumes and weights of sea cucumber measurements were obtained using a density meter (Shinko Denshi Co., Ltd., DME-220) and digital balance (A\&D Co., Ltd., ER-180A), respectively, after blotting the water on the external surface of the animals using a paper towel. The average of the actual volumes and weights were obtained after 20 replicate measurements. Using Archimedes' theory, the actual volumes were measured by applying to Eq. (1) the weights of the sea cucumbers in air and in distilled water, and the water density of the distilled water. The water density in this study was considered as 1 , because the density of pure water is $0.997 \mathrm{~g} / \mathrm{cm}^{3}$ at room temperature $\left(25^{\circ} \mathrm{C}\right)[10]$.

$$
\text { Actual volume }=\frac{\text { weight in air }[\mathrm{g}]-\text { weight in water }[\mathrm{g}]}{\text { density of water }\left[\mathrm{g} / \mathrm{cm}^{3}\right]}
$$

\subsection{Image processing to measure $B L, B W$ and $T$}

Fig. 1 shows an image-processing flowchart for measuring the $B L, B W$, and $T$ of the sea cucumber based on the vision system.

As shown in Fig. 1(a), preprocessing involved capturing images of the sea cucumber, which were projected by the backlights of the CCD cameras installed at the bottom and sides of a tank to measure the sea cucumber features. The brightness information of the black-and-white images was then converted to an 8-bit gray scale. At that point because the $B L, B W$, and $T$ should be measured after removing the sea cucumber thorns [14], 8-bit gray scale-based images were converted to $9 \times 9$ matrix structure images by performing a closing calculation using morphological image processing to partially remove the thorns in the images [21]. Finally, in the binarization process and based on the 60 level, the pixel brightness was converted to a range between 0 and 255 to improve clarity of the edge lines in the 8-bit projected bottom and side images. The base coordinate system was then configured to each output image. As shown in Fig. 1(b), to measure the $B L, B W$, and $T$ of the sea cucumber after preprocessing, the closed curve was detected from the projected bottom and side images; the center point and angles of the closed curve area were then extracted, whereby a base coordinate system was drawn.

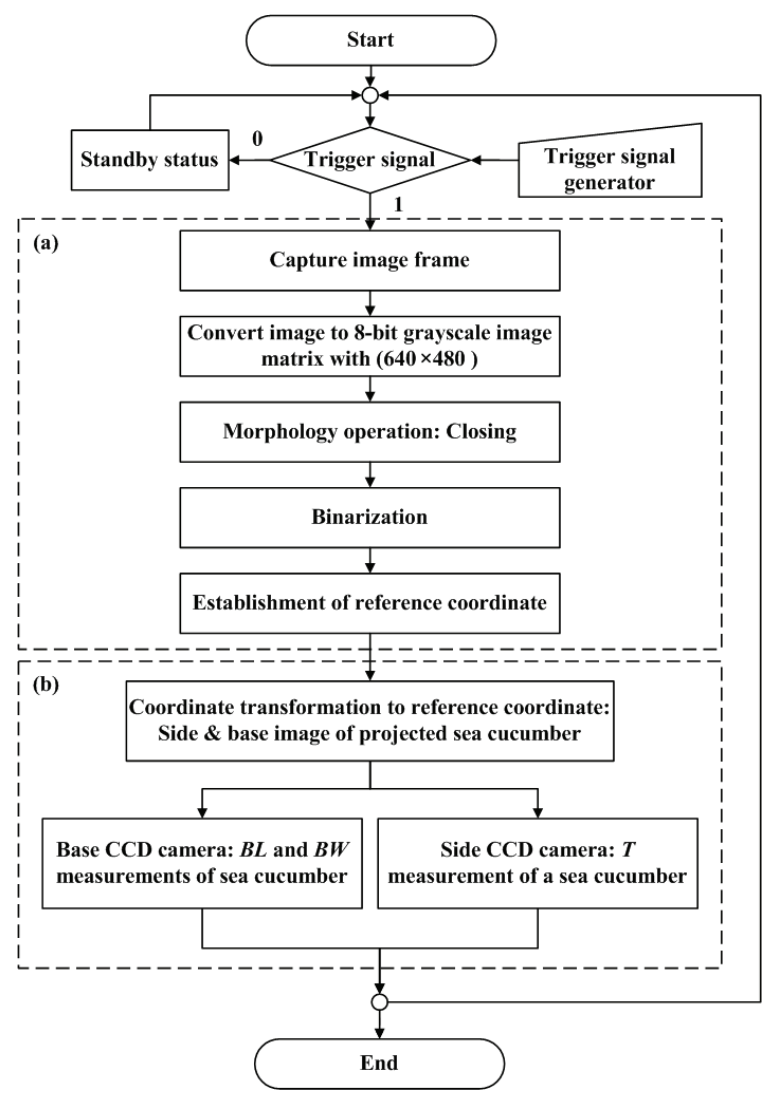

Fig. 1. Image processing flowchart: (a) preprocessing; (b) measuring process. 
Fig. 2 shows images captured while measuring the $B L$ of the sea cucumber. The $B L$ of the sea cucumber was measured from the estimated centerline in the projected bottom image. The measurement process was as follows:

1) As shown in Fig. 2(a), the circular and linear patterns were extracted from the bottom image of the sea cucumber, which was projected on the $x-y$ plane; the bottom image was scanned from the outside to the center point of the area. Then, 100 edge points were extracted.

2) Of the extracted edge points, as shown in Fig. 2(b), the left coordinate $\left(x_{0}, y_{0}\right)$ and right coordinate $\left(x_{9}, y_{9}\right)$, which were the farthest from the area's center point, were selected.

3 ) The $x$ coordinate area of the selected two points, $x_{0}-x_{9}$, was divided by eight to obtain a straight-line equation $(x$ $\left.=x_{1}, x_{2} \ldots x_{8}\right)$. Then, as shown in Fig. 2(c), the center coordinates $\left(x_{1}, y_{2}\right), \ldots\left(x_{8}, y_{8}\right)$ of the edge points, where brightness was considerably different on the straight
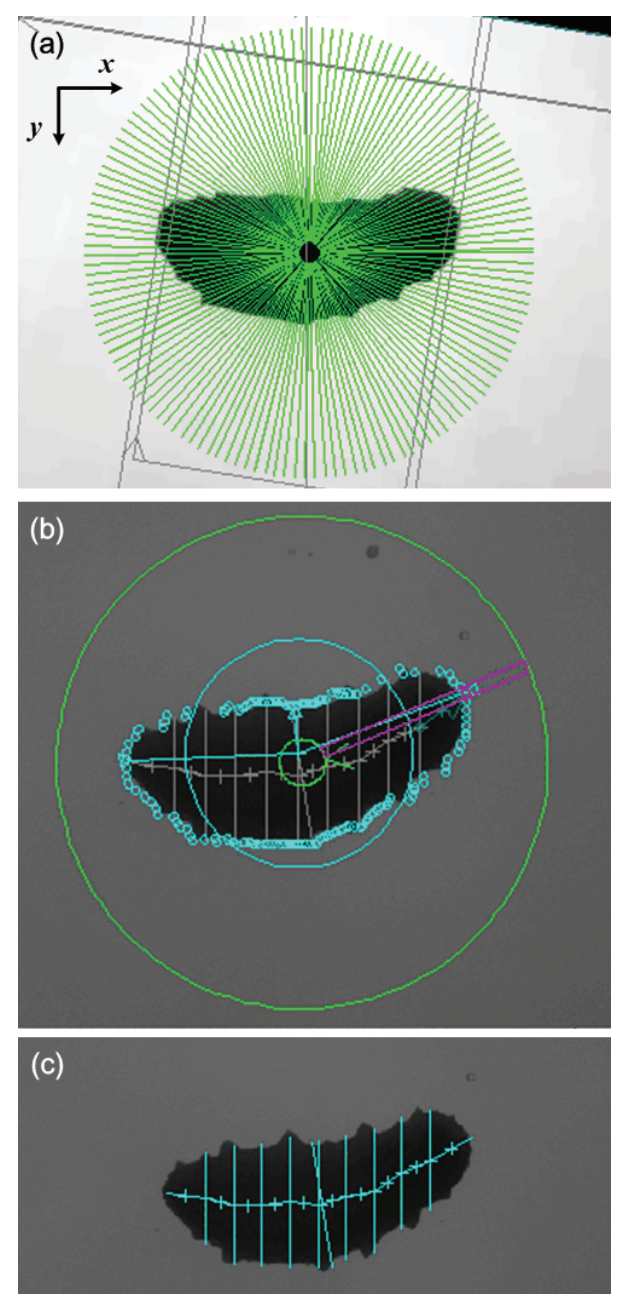

Fig. 2. Process of measuring sea cucumber $B L$ : (a) detection of edge points; (b) detection of the left and right coordinates that are the farthest from the center point; (c) connection of the detected coordinates. line, were detected.

4) The $B L$ of the sea cucumber was measured by applying each detected coordinate to Eq. (2).

$$
B L=\sum_{i=0}^{9} \sqrt{\left(x_{i+1}-x_{i}\right)^{2}+\left(y_{i+1}-y_{i}\right)^{2}}
$$

Fig. 3 shows the image captured in the sea cucumber $B W$ measurement process which was as follows:

1) As shown in Fig. 3, in reference to the center point angle in the bottom image, in which the sea cucumber was projected on the $x-y$ plane, the straight-line equation passing the center of gravity was detected.

2) The two edge points, whose brightness levels were substantially different on the straight-line were detected to measure the sea cucumber $B W$.

3) The distance between the coordinates of the two edge points was then designated as the $B W$.

Fig. 4 shows the image captured in the process of measuring the sea cucumber $T$. Fig. 5 shows the thickness errors according to measurement points observed while moving a $10 \mathrm{~mm}$ regular hexahedron standardized specimen along the $y$-axis. The measurement point was at the area center point of the sea cucumber's bottom image.

As shown in Fig. 5, measuring thickness using CCD cameras, in general, led to variations because of the perspective between the point of the measured specimen and the CCD camera installed at the side. Hence, an additional modification process was required for this study. To minimize variations when measuring $T$, the linear regression analysis between the location of the sea



Fig. 3. Measurement of sea cucumber $B W$.

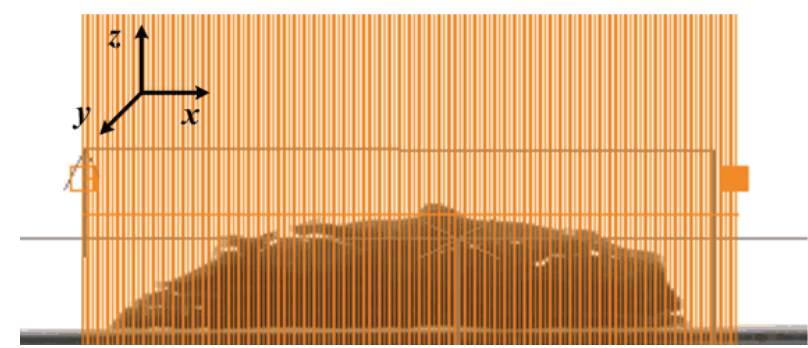

Fig. 4. Measurement of the sea cucumber $T$. 


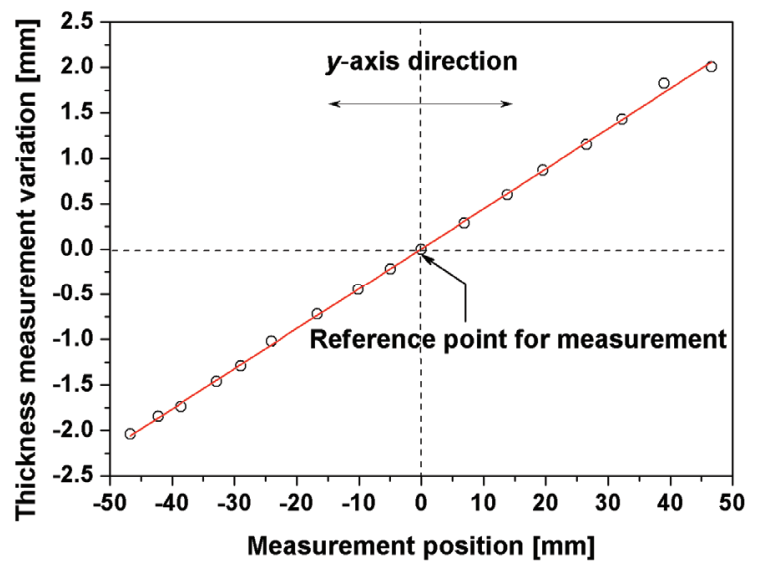

Fig. 5. Variations in thickness according to measurement points.

cucumber and the measured $T$ was performed. The revision equation was derived, as shown in Eq. (3), where $t$ is the measured thickness of the sea cucumber; $y$ is the location of the sea cucumber; and $\alpha$ and $\beta$ are regression coefficients, which were 0.044 and 0.009 , respectively.

$$
T=t-(\alpha \cdot y+\beta)
$$

The process of measuring sea cucumber $T$ was as follows:

1) As shown in Fig. 4, 100 edge points were detected in the side image. The sea cucumber was projected in the $x$-z plane by scanning the image from white to black in the direction of the center of gravity using the straightline pattern.

2) Of the detected 100 edge points, the farthest edge point from the $x-y$ plane and center of gravity - i.e., the location of the sea cucumber from the bottom image of the sea cucumber projected on the $x-y$ plane - were simultaneously detected.

3) The sea cucumber $T$ was measured by the distance between the detected edge point and $x-y$ plane, and it was calibrated as shown in Eq. (3).

The sea cucumber shrinks to a half oblate ellipsoid at its maximum as it is handled in the air. Thus, assuming that the early shape of the sea cucumber is a half oblate ellipsoid, as shown in Figs. 2, 3, and 4, the $B L, B W$, and $T$ were simultaneously measured using the vision system. They were then calculated using Eq. (4), which is a modified ellipsoid equation. The actual volume $\left(A_{v}\right)$ and calculated volume $\left(C_{v}\right)$ were compared and verified through linear regression analysis using Eq. (5), where $\alpha$ and $\beta$ are regression coefficients.

$$
\begin{gathered}
C_{v}\left[\mathrm{~cm}^{3}\right]=\frac{\pi}{6} \cdot B L \cdot B W \cdot T \\
A_{v}\left[\mathrm{~cm}^{3}\right]=\alpha \cdot C_{v}+\beta
\end{gathered}
$$

\subsection{Regression analysis for designing a sea cucumber weight estimation algorithm}

For design optimization of the sea cucumber weight estimation algorithm, regression analyses on the measured $B L, B W, T$, and $A_{v}$, and on the $C_{v}$ were performed based on the assumption that the sea cucumber form is a half oblate ellipsoid. The $R^{2}$ and regression equation were then obtained. The power curve regression model was used for the regression analyses of the $B L \mathrm{~s}, B W \mathrm{~s}$, and $T \mathrm{~s}$ against the weights; the linear regression model was used for the actual sea cucumber volumes against the weights.

\subsection{Experimental systems}

We produced a vision system-based device (size: $600 \times$ $500 \times 1300 \mathrm{~mm}$ ) for measuring sea cucumber features, $B L \mathrm{~s}, B W \mathrm{~s}, T \mathrm{~s}$, and volumes, as shown in Fig. 6 . The vision system (PV-200, Panasonic) and CCD cameras were installed at the bottom and sides, respectively, to capture two-dimensional images of the bottom and sides of the sea cucumber. These images were projected on the twodimensional plane. In the opposite direction of each CCD camera, a $30 \mathrm{~W}$ infrared backlight was installed to generate a silhouette of the sea cucumber. A data collection device (National Instruments, cDAQ-9174) was installed to collect the visual information required to measure the $B L$, $B W$, and $T$ and to calculate the volume, based on LabVIEW software (version: LabVIEW 2013, National Instruments).

Fig. 7 shows the single slide rail system for measuring sea cucumber weights that we produced in a laboratory

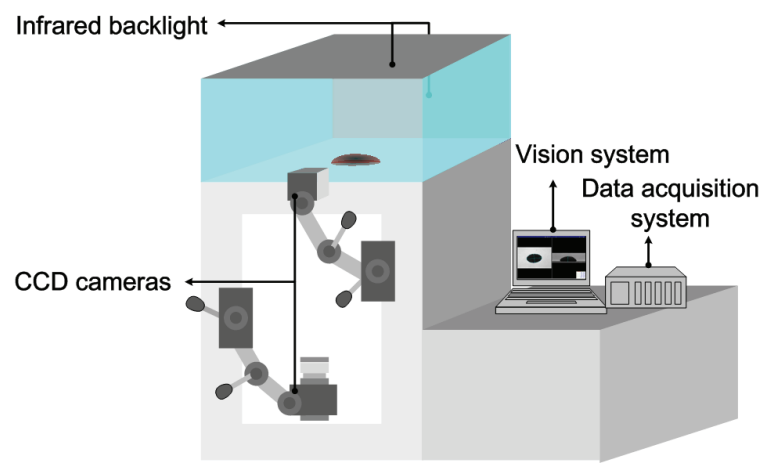

Fig. 6. Device for measuring sea cucumber features.

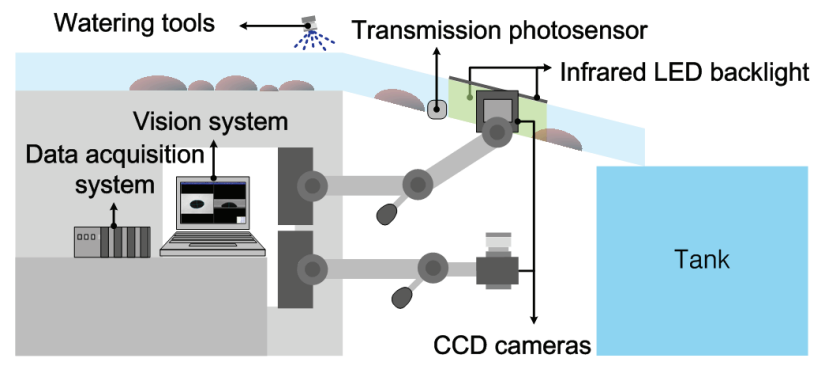

Fig. 7. Single slide rail device for measuring sea cucumber weights. 
size to verify the performance of the optimized sea cucumber weight estimation algorithm through repeated testing. The system components were the same as those of the sea cucumber feature measurement device, except for the addition of an installed photosensor (Autonics Co., LTD, BYS500-TDT) that generates trigger signals to automatically capture images. The weight of each sea cucumber whose respiratory tree was emptied of water in advance was measured by dropping it in the single side rail linked to the tank. At that point, the optimized algorithm for sea cucumber's weight estimation was used to continuously measure the weights of sea cucumbers moving in the rail.

\section{Results and Discussion}

\subsection{Repeatability of measurement}

The inspection or measurement method of a certain object based on images leads to respective inspection or measurement errors depending on the brightness of lighting and external environments. Therefore, the repeatability of the designed algorithm for sea cucumber features was measured to verify its reliability. After selecting standardized regular hexahedron specimens with a regular surface (dimensions: $10 \times 10 \times 10 \mathrm{~mm}$ ), their lengths, widths, and heights were simultaneously measured 300 times within an interval of a second under the same measurement conditions.

Fig. 8 shows the results of 300 measurements of the standardized regular hexahedron specimens. Table 1 indicates the mean, standard deviation, and coefficient of variation of the features. As a result, the means,

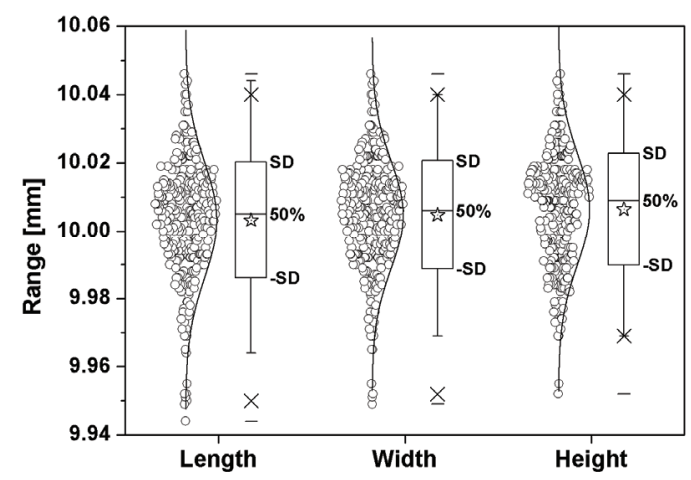

Fig. 8. Test results of repeated measurements of the standardized regular hexahedron specimens.

Table 1. Results of measurements: mean, standard deviation, and coefficient of variation

\begin{tabular}{c|c|c|c}
\hline Features set & Mean $[\mathrm{mm}]$ & SD $[\mathrm{mm}]$ & CV [\%] \\
\hline Length & 10.002 & 0.017 & 0.170 \\
\hline Width & 10.003 & 0.016 & 0.160 \\
\hline Height & 10.004 & 0.016 & 0.160 \\
\hline
\end{tabular}

standard deviations, and coefficients of variation were within $10.004 \mathrm{~mm}, 0.017 \mathrm{~mm}$, and $0.170 \%$, respectively, thereby confirming high repeatability.

\subsection{Design of sea cucumber weight estimation algorithm}

For optimized design of the sea cucumber weight estimation algorithm, regression analyses were performed on the $B L \mathrm{~s}, B W \mathrm{~s}, T \mathrm{~s}$, actual volumes, and calculated volumes - where sea cucumber form was assumed to be a half oblate ellipsoid - against the sea cucumber weights.

Figs. 9 through 11 show the correlations of the $B L, B W$, and $T$ against the weight. Each correlation was nonlinearly distributed. The power curve regression model was used to examine the regression equation and $R^{2}$ value, as shown in Table 2. Here, $y$ denotes weight, $x$ represents the measurement item, and $\alpha$ and $\beta$ are regression coefficients. The test results confirmed that the $R^{2}$ values of the $B L, B W$, and $T$ against the weight of the sea cucumber were all

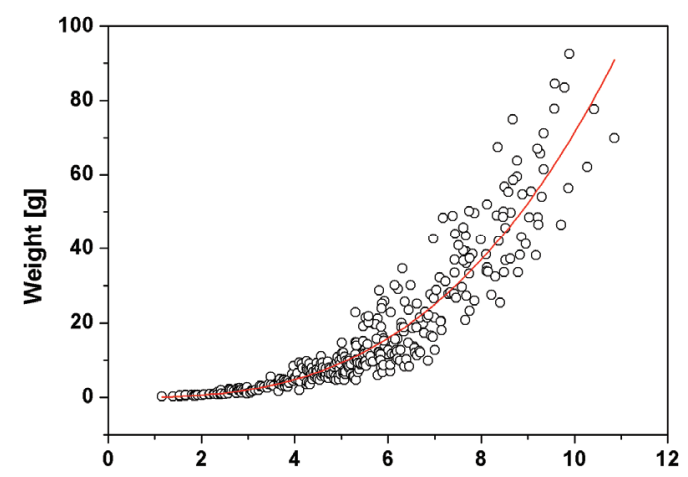

Fig. 9. Plot of power curve fit of weight compared with $B L$ and weight.



Fig. 10. Plot of power curve fit of weight compared with $B W$ and weight.

Table 2. Equation fit result for sea cucumber weight compared to $B L, B W$, and $T$

\begin{tabular}{c|c|c|c}
\hline Equation: $y=\alpha \cdot x^{\beta}$ & $B L$ & $B W$ & $T$ \\
\hline$\alpha$ & 0.081 & 1.876 & 4.135 \\
\hline$\beta$ & 2.945 & 2.154 & 2.694 \\
\hline$R^{2}$ & 0.871 & 0.904 & 0.925 \\
\hline
\end{tabular}


$>0.871$, where the $R^{2}$ value between the $T$ and weight was rather high (0.925).

However, Figs. 9, 10, and 11 suggest that the smaller size of the sea cucumber indicates a smaller weight deviation. Thus, the larger the size of the sea cucumber is, the bigger the weight deviation is, implying that a bigger error would occur in estimating the weight of a large sea cucumber. In addition, the weight against the $B L, B W$, and $T$ was nonlinear; therefore, a more precise measurement technology would be required when classifying sea

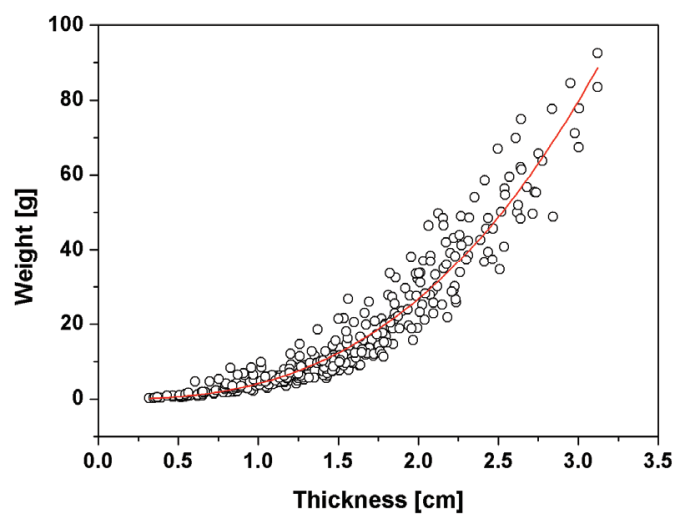

Fig. 11. Plot of power curve fit of weight compared with $T$ and weight.

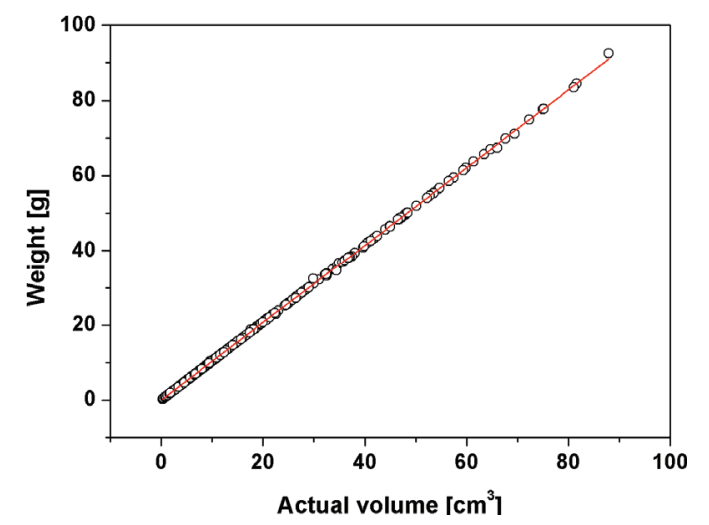

Fig. 12. Plot of linear fit of weight compared with $A_{v}$ and weight.

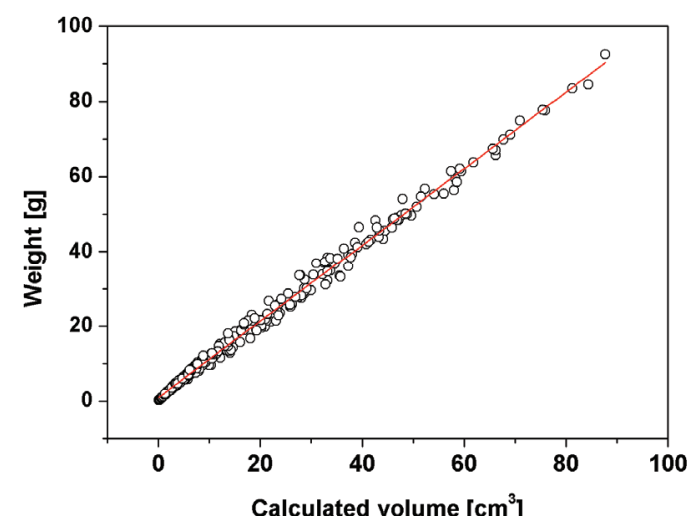

Fig. 13. Plot of linear fit of weight compared with $C_{v}$ and weight. cucumbers according to their weights.

Figs. 12 and 13 show the correlations between the actual volume and calculated volume where the sea cucumber was assumed to be a half oblate ellipsoid. Each correlation was linearly distributed. The regression equation and $R^{2}$ value were investigated using the linear regression model, as shown in Table 3 .

The $R^{2}$ value between the actual volume and weight was 0.999 , which was relatively very high. The $R^{2}$ value between the calculated volume and weight was 0.995 , which was likewise high. The results of comparing each regression equation confirmed that the differences in the slope and $y$-intercept were approximately 0.017 and 0.944 , respectively.

As shown in Fig. 14, we ran a linear regression analysis using Eq. (5) to compare and verify the correlation between the actual volumes and calculated volumes.

The coefficients of variation $\alpha$ and $\beta$ were 0.984 and 0.912 , respectively, where the $R^{2}$ value was 0.995 . The regression analysis, where $\beta$ was 0 , showed $\alpha$ and $R^{2}$ were 1.010 and 0.997 , respectively. Thus, the regression equation slope and $R^{2}$ between the actual volume and calculated volume were both close to 1 . Moreover, the $R^{2}$ value was also high. Based on this finding, we confirmed that there was no significant difference between the actual volume and the calculated volume in experimental range.

The results of performing the regression analyses of the $B L, B W, T, A_{v}$, and $C_{v}$ against weight confirmed that the method of measuring the actual sea cucumber volume was effective in estimating sea cucumber weight. Therefore, using the regression equation between the actual volume and weight, as shown in Table 3 and Eqs. (4) and (5), and the algorithm - which estimates sea cucumber weight by measuring its volume (Eq. (6), where $C_{1}$ and $C_{2}$ denote

Table 3. Equation fit result for sea cucumber weight compared to $A_{v}$ and $C_{v}$

\begin{tabular}{c|c|c}
\hline Equation: $\mathrm{y}=\alpha \cdot x+\beta$ & $A_{v}$ & $C_{v}$ \\
\hline$\alpha$ & 1.036 & 1.019 \\
\hline$\beta$ & 0.013 & 0.957 \\
\hline$R^{2}$ & 0.999 & 0.995 \\
\hline
\end{tabular}

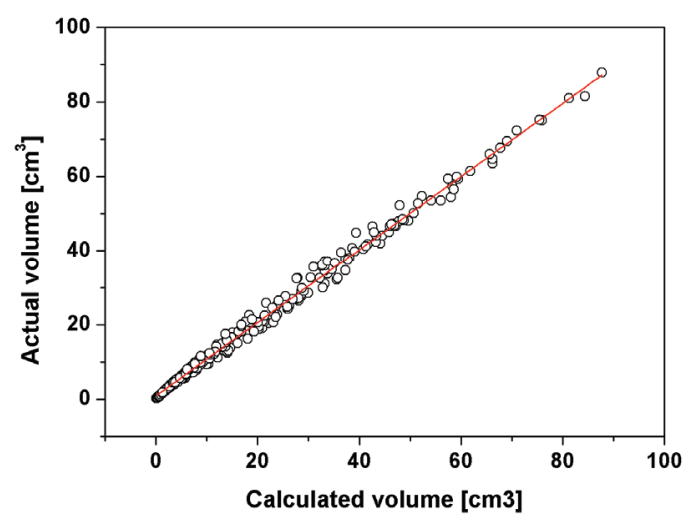

Fig. 14. Plot of linear fit of weight compared with $C_{v}$ and $A_{v}$. 


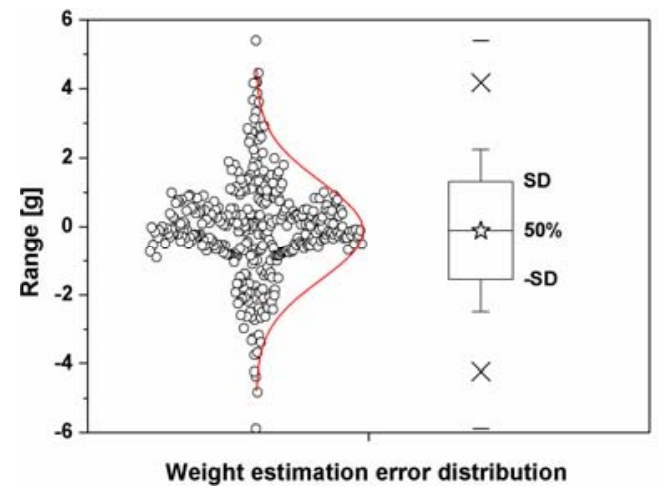

Fig. 15. Sea cucumber weight prediction error distribution, evaluated by leave-on out cross-validation.

the constants calculated with the regression coefficients between the actual volume and weight and between the actual and calculated volume) - the $C_{1}$ and $C_{2}$ of our experiments were determined to be 0.534 and 0.958 , respectively.

$$
\text { Weight }[g]=C_{1} \cdot(B L \cdot B W \cdot T)+C_{2}
$$

The performance of this algorithm was evaluated through leave-one-out cross-validation [22, 23], moreover, as shown in Fig. 15, the root mean square error and weight estimation error spread were $1.434 \mathrm{~g}$ and $\pm 5.879 \mathrm{~g}$, respectively. These finding prove that the accuracy of this algorithm improved compared to that of existing estimation methods that measure three-dimensional projected volume of the fish [24, 25]. It was confirmed that the developed weight estimation algorithm and single slide rail system for weight measurement can weigh approximately 4,500 sea cucumbers per hour.

\section{Conclusion}

Regression analysis between each feature and the weight of the sea cucumber to estimate weight showed that the correlation between the actual volume and weight of the sea cucumber was high. The proposed algorithm was shown to estimate the weight of sea cucumbers by measuring their volumes using the vision system. This method calculated the volume of the sea cucumber by assuming its early form as a half oblate ellipsoid; moreover, it proved to be more convenient than existing measurement methods in which the line laser and vision system were combined. Moreover, optimization was performed through linear regression analysis between the calculated and actual volume of the sea cucumber.

The developed sea cucumber weight estimation algorithm can rapidly and accurately measure the growth of sea cucumbers that are raised within the recently developed integrated multi-trophic aquaculture (IMTA) system; facilitates automated management. The proposed algorithm shows potential applications to the selection of sea cucumbers.

\section{Acknowledgements}

This work was supported by a grant (RP-2014-FE-013) from the National Fisheries Research and Development Institute (NFRDI), Republic of Korea.

\section{References}

[1] T. W. Fulton, The Rate of Growth of Fishers. Edinburgh: Fisheries Board Scotland, Edinburgh, 1904, pp. 141-241.

[2] M. Y. Ibrahim and J. Wang, "Mechatronics applications to fish sorting — part 1: fish size identification," IEEE International Symposium on Industrial Electronics, pp. 1978-1983, 2009.

[3] S. J. Jeong, Y. S. Yang, K. Lee, J. G. Kang, and D. G. Lee, "Vision-based automatic system for non-contact measurement of morphometric characteristics of flatfish," Journal of Electrical Engineering \& Technology, vol. 8, pp. 1194-1201, 2013.

[4] N. J. C. Strachan, "Sea trials of a computer vision based fish species sorting and size grading machine," Mechatronics, vol. 4, pp. 773-783, 1994.

[5] D. J. White, C. Svellingen, and N. J. C. Strachan, "Automated measurement of species and length of fish by computer vision," Fish. Res., vol. 80, pp. 203-210, 2006.

[6] M. O. Balaban, G. F. Ünal Şengör, M. G. Soriano, and E. G. Ruiz, "Using image analysis to predict the weight of Alaskan salmon of different species," J. Food Sci., vol. 75, pp. E157-E162, 2010a.

[7] M. O. Balaban, M. Chombeau, D. Cirban, and B. Gümüş, "Prediction of the weight of Alaskan pollock using image analysis," J. Food Sci., vol. 75, pp. E552E556, $2010 \mathrm{~b}$.

[8] D. J. Lee, R. M. Lane, and G. H. Chang, "Threedimensional reconstruction for high-speed volume measurement," Intelligent Systems and Smart Manufacturing. International Society for Optics and Photonics, 2001.

[9] D.J. Lee, J. Eifert, P. Zhan, and B. Westover, "Fast surface approximation for volume and surface area measurements using distance transform," Opt. Eng., vol. 42, pp. 2947-2955, 2003.

[10] S. Damar, Y. Yagiz, M.O. Balaban, S. Ural, A. C. M. Oliveira, and C.A. Crapo, "Prediction of oyster volume and weight using machine vision," J. Aquat. Food Prod. Technol., vol. 15, pp. 3-15, 2007.

[11] S. Feindel, T. Bennett, and K. Kanwit, "The Maine sea cucumber (Cucumaria frondosa) fishery," Submitted to Standing Legislative Committee on Marine 
Resources. Department of Marine Resources, Maine, http://www.maine.gov/dmr/cukes/feinde/eta/2011.pdf, 2011.

[12] S. Choe and Y. Ohshima, "On the morphological and ecological differences between two commercial forms. 'green' and 'red', of the Japanese common sea cucumber, Stichopus japonicas Selenka," Nippon Suisan Gakkaishi, vol. 27, pp. 97-106, 1961.

[13] B. Gümüş and M. O. Balaban, "Prediction of the weight of aquacultured rainbow trout (Oncorhynchus mykiss) by image analysis," J. Aquat. Food Prod. Technol., vol. 19, pp. 227-237, 2010.

[14] Y. Yamana and T. Hamano, "New size measurement for the Japanese sea cucumber Apostichopus japonicas (Stichopodidae) estimated from the body length and body breadth," Fish. Sci., vol.72, pp.585-589, 2006.

[15] J. Y. Yingst, "Factors influencing rates of sediment ingestion by Parastichopus parvimensis (Clark), an epibenthic deposit-feeding holothurians," Estuar. Coast. Shelf Sci., vol. 14, pp. 119-134, 1982.

[16] K. Mitsukuri, "Notes on the habits and life-history of Stichopus japonicus Selenka," 1903.

[17] Y. W. Dong, S. L. Dong, X. L. Tian, F. Wang, and M. Z. Zhang, "Effects of diel temperature fluctuations on growth, oxygen consumption and proximate body composition in the sea cucumber Apostichopus japonicus Selenka," Aquaculture, vol. 255, pp. 514521, 2006.

[18] L. N. Zamora and A. G. Jeffs, "Feeding metabolism and growth in response to temperature in juveniles of the Australasian sea cucumber, Australostichopus mollis," Aquaculture, vol. 15, pp. 358-359, 2012.

[19] T. Ji, Y. Dong, and S. Dong, "Growth and physiological responses in the sea cucumber, Apostichopus japonicus Selenka: aestivation and temperature," Aquaculture, vol. 283, pp. 180-187, 2008.

[20] D. L. Paltzat, C. M. Pearce, P. A. Barnes, and R. S. McKinley, "Growth and production of California sea cucumbers (Parastichopus californicus Stimpson) cocultured with suspended Pacific oysters (Crassostrea gigas Thunberg)," Aquaculture, vol. 275, pp. 124-137, 2008.

[21] R. Gonzalez and R. Woods, Digital Image Processing, New Jersey: Prentice Hall, 2007.

[22] P. A. Lachenbruch and R. M. Mickey, "Estimation of error rates in discriminant analysis," Technometrics, vol. 10, pp. 1-11, 1968.

[23] S. Theodoridis and K. Koutroumbas, Pattern recognition, San Diego, California: Academic Press, 1999.

[24] J. R. Mathiassen, E. Misimi, B. Toldnes, M. Bondø, and S. O. Østvik, "High-speed weight estimation of whole herring (Clupea harengus) using 3D machine vision," J. Food Sci., vol. 76, pp. E458-E464, 2011.

[25] F. Stobeck and B. Daan, "Weight estimation of flatfish by means of structured light and image analysis," Fish. Res., vol. 11, pp. 99-108, 1991.

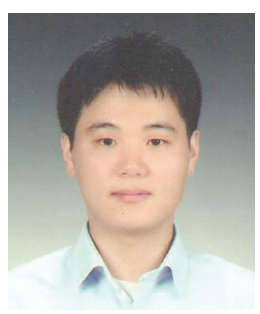

Donggil Lee received B.S., M.S. and $\mathrm{Ph} . \mathrm{D}$. degree in Electrical Engineering from Pusan National University in 2008, 2010 and 2013, respectively. He has been a senior researcher in NFRDI, Busan, Korea. His research interests are in the field of vision algorithm, machine vision, automatic control system design, circuit design, and robotics.

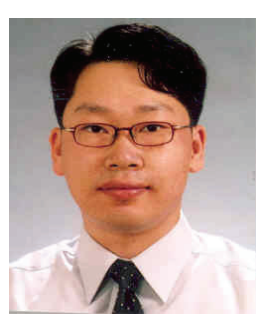

Seonghun Kim received his BE and ME degree in Fisheries Physics in Pukyong National University. in 2000 and 2002, respectively and his Ph.D. degree in Fisheries Engineering from Hokkaido Univ. in 2008. He is a researcher at Fisheries system engineering Division, NFRDI, busan, Korea.

His research interests include Fishing gear design, mesh size selectivity, echo-friendly fishing gear.

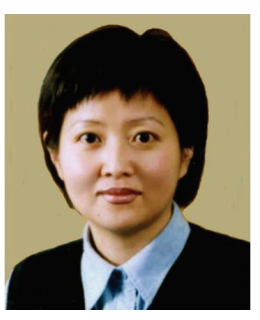

Miseon Park received her Ph.D. in Shellfish Disease from Pukyong National University in 1989. Since April 1982, she has been a junior researcher, a senior researcher, and a director general in NFRDI, Busan, Korea. Her research interests include shellfish aquaculture, shellfish disease, and Integrated Multi-Trophic Aquaculture.

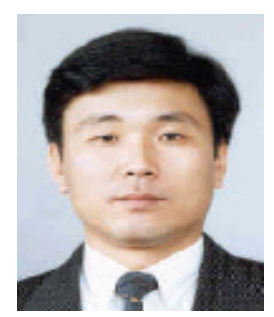

Yongsu Yang received his Ph.D. degree in Fishery Engineering from Jeju National University in 2000. He has been a senior researcher in NFRDI (National Fisheries Research \& Development Institute), Busan, Korea. His research interest is the fishing gear design, and underwater acoustics. 